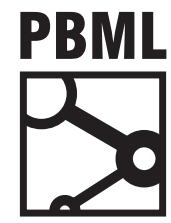

The Prague Bulletin of Mathematical Linguistics

NUMBER 108 JUNE 2017 331-342

\title{
Linguistically Motivated Vocabulary Reduction for Neural Machine Translation from Turkish to English
}

\author{
Duygu Ataman, ${ }^{\mathrm{ab}}$ Matteo Negri, ${ }^{\mathrm{b}}$ Marco Turchi, ${ }^{\mathrm{b}}$ Marcello Federico ${ }^{\mathrm{b}}$ \\ a Università degli Studi di Trento, Trento, Italy \\ b Fondazione Bruno Kessler, Trento, Italy
}

\begin{abstract}
The necessity of using a fixed-size word vocabulary in order to control the model complexity in state-of-the-art neural machine translation (NMT) systems is an important bottleneck on performance, especially for morphologically rich languages. Conventional methods that aim to overcome this problem by using sub-word or character-level representations solely rely on statistics and disregard the linguistic properties of words, which leads to interruptions in the word structure and causes semantic and syntactic losses. In this paper, we propose a new vocabulary reduction method for NMT, which can reduce the vocabulary of a given input corpus at any rate while also considering the morphological properties of the language. Our method is based on unsupervised morphology learning and can be, in principle, used for pre-processing any language pair. We also present an alternative word segmentation method based on supervised morphological analysis, which aids us in measuring the accuracy of our model. We evaluate our method in Turkish-to-English NMT task where the input language is morphologically rich and agglutinative. We analyze different representation methods in terms of translation accuracy as well as the semantic and syntactic properties of the generated output. Our method obtains a significant improvement of 2.3 BLEU points over the conventional vocabulary reduction technique, showing that it can provide better accuracy in open vocabulary translation of morphologically rich languages.
\end{abstract}

\section{Introduction}

Neural machine translation (NMT) is a recent approach to machine translation (MT), which exploits deep learning to directly model the translation probability of 


\begin{tabular}{ll}
\hline Turkish & English \\
\hline \hline duy(-mak) & (to) sense \\
duygu & sensation \\
duygusal & sensitive \\
duygusallaş(-mak) & $($ to become sensitive \\
duygusallaştırıl(-mak) & $($ to $)$ be made sensitive \\
duygusallaştırılmış & the one who has been made sensitive \\
duygusallaştırılamamış & the one who could not have been made sensitive \\
duygusallaştırılamamişlardan & from the ones who could not have been made sensitive \\
\hline
\end{tabular}

Table 1. Turkish-to-English translation

texts in two different languages. Although the first models (Sutskever et al., 2014; Bahdanau et al., 2014) are only few years old, today NMT has already become the new state-of-the-art. Similar to other statistical approaches to MT, NMT is an instance of supervised learning, where a probabilistic model learns to predict an output given the input, based on an history of translation examples. The accuracy of the model is limited by the ability of the system to generalize to unseen examples, which is still an open issue in NMT due to computational restrictions. Current implementations of the model are computationally expensive; they require huge amounts of training time and memory space due to the large number of parameters to optimize. The translation engine uses a word vocabulary whose size is limited in order to control the complexity of the model. However, a text can only be translated if an exact match of the given source word can be found in the vocabulary.

Data sparseness, especially due to rare content words or infrequent inflected word forms, is one of the main reasons that limits the current performance of NMT in lowresourced and morphologically rich languages. For instance, Turkish, the language we focus on in this paper, is an agglutinative language where morphological inflections occur through attachment of suffixes to a given stem. Most syntactic forms in English, such as prepositions, negation, person or copula, are achieved solely through morphological inflections in Turkish. Table 1 illustrates the distance from Turkish to English in terms of the required translations to be generated by an ideal MT system. There are about 30,000 root words and 150 distinct suffixes in Turkish, which can experience agglutinative concatenations and internal changes through fusion to achieve vowel harmony, and cause the morphological tags to grow exponentially (Oflazer and El-Kahlout, 2007). Hence, the search for alternative word representation techniques that can solve the sparsity problem in Turkish is extremely important and can allow better handling of the input complexity.

Recent studies have tried implicitly extending the vocabulary by segmenting the words in the corpus into smaller units such as characters (Ling et al., 2015; Lee et al., 2016), sub-words (Sennrich et al., 2016; Wu et al., 2016) or hybrid (Luong and Manning, 
2016) units. The problem with these approaches is that they disregard any notion of morphology during estimation of the sub-word units, which may lead to loss of semantic and syntactic information preserved in the word structure. In this paper, we propose to overcome this problem by developing a linguistically motivated segmentation method for open vocabulary translation of morphologically rich languages. We present a novel method that can perform segmentation to fit any desired vocabulary size for NMT while also considering the morphological properties of words. Being unsupervised, the proposed method can be fundamentally used with any language pair and direction in MT. We evaluate the benefit of our approach in a Turkish-toEnglish (TR-EN) NMT task against a conventional vocabulary reduction method that relies solely on statistics, and a supervised method that applies segmentation based on morphological analysis. The results show that our linguistically motivated vocabulary reduction method achieves significantly better translation accuracy compared to the conventional method and maintains its performance at different rates of vocabulary reduction.

\section{Neural Machine Translation}

The NMT model we use in this paper is based on the encoder-decoder and attention models described in (Bahdanau et al., 2014). First, a bi-directional RNN (the encoder) maps the sparse one-hot representation of an input sentence $X=\left(x_{1}, x_{2}, \ldots x_{m}\right)$ into corresponding dense vectors called encoder hidden states. Then, a unidirectional RNN (the decoder) step-wisely predicts the target sequence $Y=\left(y_{1}, y_{2}, \ldots y_{j} \ldots y_{l}\right)$ as follows. The $i^{\text {th }}$ target word is predicted by sampling from a word distribution computed from the previous target word $y_{i-1}$, the previous hidden state of the decoder, and a convex combination of the encoder hidden states (i.e. context vector). In particular, each weight of the combination is predicted by the attention model, on the basis of the previous target word, the previous decoder hidden state and the corresponding encoder hidden state. Both the encoder and decoder RNNs are implemented with GRU gates (Cho et al., 2014). The dimensions of the embeddings and hidden layers are proportional to the vocabulary size. Large vocabularies hence imply more parameters and higher computational costs.

\section{Related Work}

In general, two approaches have been proposed to cope with the limited vocabulary problem in NMT. The first one includes purely statistical methods, which aim to predict a set of sub-words that can optimally fit a given vocabulary size. These methods achieved state-of-the-art results for many morphologically rich languages (e.g. German, Russian, Czech and Finnish).

One such method is Byte-Pair Encoding (BPE), a likelihood-based sub-word unit generation method. BPE is originally a data compression algorithm (Gage, 1994), and 


\begin{tabular}{lll}
\hline Corpus Frequency & Vocabulary Entry & English Translation \\
\hline \hline 1011 & hapishane & jailhouse \\
793 & hapishan@@ & - \\
587 & hapishanede & in the jailhouse \\
245 & hapishaneden & from the jailhouse \\
229 & hapishanesinde & at the jailhouse of $($ him $/$ her $/$ it) \\
181 & hapishanenin & of the jailhouse \\
100 & hapishanesine & to the jailhouse of $(\mathrm{him} / \mathrm{her} / \mathrm{it})$ \\
\hline
\end{tabular}

Table 2. Turkish vocabulary entries obtained with BPE

\begin{tabular}{llll}
\hline Source & Segmentation & NMT Output & Reference \\
\hline \hline kanunda & kan@@ unda & in your blood & in the law \\
\hline sigortalılar & sigor@@ talı@@ lar & the insurers & the insured ones \\
\hline
\end{tabular}

Table 3. Translation examples obtained when BPE is applied on Turkish words

has been recently modified by Sennrich et al. (2016) for vocabulary reduction, where the most frequent character sequences are iteratively merged to find the optimal description of the corpus vocabulary. Open vocabulary translation using this method is based on the assumption that many types of words can be translated when segmented into smaller units, such as named entities, compound words, and loanwords (Sennrich et al., 2016). Nevertheless, in cases of common morphological paradigms such as the derivational or inflectional transformations which are typically observed in Turkish, the method lacks a linguistic notion which would allow it to better generalize syntactic patterns among the data and use the vocabulary space more effectively. Table 2 lists some of the entries found in the NMT dictionary after the segmentation of the corpus with BPE, which stores many repetitions of the same lemma in different surface forms, indicating an inefficacy in capturing a compact representation of the data. Another crucial problem is related to the semantic losses which occur due to segmenting words at positions which breaks the morphological structure. Table $\underline{3}$ presents some of the typical mistakes observed in the NMT output when BPE is applied for segmentation. In the first example, the Turkish word kanunda (translation: in the law), the lemma of which is kanun (translation: law), is segmented in the middle of the root, which causes a semantic shift. The segmented word now becomes a completely different word, kan (translation: blood). In the second example, segmentation of the suffixes leads to generate the wrong inflected form in English.

Another set of purely statistical methods that attempted to cope with the vocabulary problem in NMT are based on the idea of constructing the translation model directly at the character-level (Ling et al., 2015; Lee et al., 2016). These models use 
deep neural networks as compositional functions to predict representations of characters and new morphological forms. However, these models also assume that, by solely relying on statistics we might be able to capture the morphological rules that form the basics of semantics and syntax of language. Moreover, these models are known to generate spurious words that do not exist in the language (Lee et al., 2016).

The second family of approaches includes methods that also consider the morphological properties of words but can only reduce the vocabulary to a limited extent, usually by applying cut-off thresholds on the vocabulary and reducing the coverage of the long tail of less frequent words. For instance, Sánchez-Cartagena and Toral (2016) have used a morphological analyzer to separate words into root and inflection boundaries to achieve vocabulary reduction for NMT. However, in addition to failing to capture a full morphological description of words (i.e. generating the complete set of affixes existent in a word), their method cannot reduce the vocabulary of a given text to fit any vocabulary size. Another study tried to overcome this limitation by using the Baseline variant of Morfessor (Creutz and Lagus, 2005b), which allows to reach a vocabulary size set prior to segmentation (Bradbury and Socher, 2016). Although providing a sense of morphology into the segmentation process, this tool neglects the morphological varieties between sub-word units, which might result in sub-word units that are semantically ambiguous (i.e. either stems or suffixes).

In conclusion, to our knowledge, there is no vocabulary reduction method for NMT that can both reduce the vocabulary size at any given rate while also considering the individual morphological properties of the generated sub-word units. We aim to solve this problem with the segmentation method described in the next section.

\section{Linguistically Motivated Vocabulary Reduction}

We present a linguistically motivated segmentation method that achieves open vocabulary translation while considering the morphological properties of individual sub-word units. First, we propose using a supervised segmentation method based on morphological analysis, which helps us to evaluate our vocabulary reduction technique in terms of its ability to generalize the morphology of language from input data. This method aims to represent words in a less sparse way while preserving the complete morphological information. Later, we describe the method proposed in this paper, an unsupervised morphology learning algorithm that predicts the sub-word units in a corpus by a prior morphology model while reducing the vocabulary size to fit a given constraint.

\subsection{Supervised Morphological Segmentation}

As a supervised approach to linguistically motivated segmentation, we use a method which can reduce the word vocabulary of the Turkish corpus to only the root words along with a set of suffix units that are represented in terms of their inflec- 
tional roles. This representation maintains a full description of the morphological properties of sub-word units in a word while minimizing the sparseness caused by inflection and allomorphy. We adopt the pre-processing approach of Bisazza and Federico (2009), who used the suffix combinatory finite-state analyzer of Oflazer (1994) to tag each sub-word unit in a Turkish word, and a morphological disambiguation tool (Sak et al., 2007) to decrease the sparseness caused by suffix allomorphy. After the pre-processing, we separate all roots and suffix tags into separate tokens and add an end-of-word (EOW) symbol for each analyzed word.

\subsection{Unsupervised morphological segmentation}

Supervised methods can provide the best accuracy in analysis, although, an ideal approach for MT should not require language-specific resources. Therefore, in this paper, we suggest to extend the unsupervised morphology induction framework Morfessor to develop a novel linguistically motivated vocabulary reduction method in NMT, which optimizes the complexity of the segmentation model with a constraint on the vocabulary size. The analysis of Creutz and Lagus (2005a) shows that Morfessor models optimized with the Maximum A-Posteriori (MAP) criterion generally achieve the best results. Our model is based on Morfessor Flatcat (Grönroos et al., 2014), a variant of this model family that uses a category-based Hidden Markov Model (HMM) and a flat lexicon structure. The category-based model is essential for a linguistically motivated segmentation as words would only be split considering the possible categories of their sub-words, preventing to split the words at random positions when a frequent sub-word is observed.

The aim of MAP optimization is to avoid overfitting by finding a balance between model accuracy and complexity. The model consists of two parts, a morpheme lexicon and a grammar that combines the language units together and generates new words. The MAP estimate of the overall system is given as:

$$
M^{*}=\operatorname{argmax}_{M} P(D \mid M) P(M)
$$

where the two factors represent the likelihood of the training corpus $D$ and the prior probability of the model $M$. The former is estimated by an HMM which considers transitions between different morpheme categories (e.g. stem to suffix) when a word is constructed. The latter is modeled considering individual properties of the generated morphemes $\mu_{i}$ :

$$
\mathrm{P}(M) \approx \mathrm{m} ! \prod_{i}^{\mathrm{m}} \mathrm{P}\left(\operatorname{usage}\left(\mu_{i}\right)\right) \mathrm{P}\left(\operatorname{form}\left(\mu_{i}\right)\right)
$$

where $m$ is the number of distinct morphemes in the lexicon (Creutz and Lagus, 2007). The usage of a morpheme is related to its meaning and is modeled with its frequency, length, and the left and rightward perplexities. The form of a morpheme is the set of physical properties that distinguish it from the others in the lexicon. 
Using the a-posteriori probability, one can train a segmentation model considering both the model complexity and the maximum-likelihood of the corpus, without any control on the size of the output lexicon. In order to use the model to achieve controlled vocabulary reduction for NMT, we insert a constraint on the desired lexicon size into the MAP optimization by applying a regularization weight over the lexicon cost and giving more favor in a reduction of the model complexity during optimization. The cost function is then estimated by the general formula:

$$
\mathrm{L}(\mathrm{D}, \mathrm{M})=-\log \mathrm{P}(\mathrm{D} \mid \mathrm{M})-\alpha \log \mathrm{P}(\mathrm{M})
$$

where a higher $\alpha$ would force the algorithm to generate a smaller lexicon size and a higher amount of segmentation. Considering the tendency of the flat lexicon models to keep the frequent words unsegmented in the corpus (Grönroos et al., 2014), in order to achieve a more accurate segmentation model we disregard the frequency distribution $\mathrm{P}\left(\mu_{i}\right)$ from the weighted part of the cost function. In fact, the value of the term is generally too small to affect the model complexity, but has an important role in determining the characteristics of the discovered morphemes.

For a given NMT vocabulary size limit, by setting the regularization weight $\alpha$ as $\frac{m_{1}}{m_{2}}$, where $m_{1}$ is the initial vocabulary size of the corpus, and $m_{2}$ is the desired vocabulary size, we achieve the right amount of regularization and the output lexicon size. The modified model has a new input parameter, output lexicon size, which sets the amount of regularization that reduces the vocabulary to the desired size. By using the parameter as a convergence limit we also minimize the model convergence time.

\section{Experimental Set-up}

We design two sets of experiments in order to evaluate our method. In the first experiment, we evaluate its ability to capture the morphological properties of sub-word units. As an indicator of vocabulary reduction that maintains the full morphological description and semantics of the original word, we deploy the supervised segmentation described in Section 4.1. However, the supervised method can only reduce the

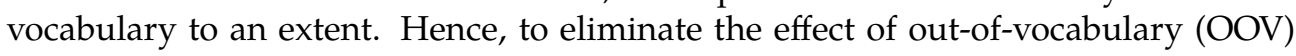
words in test set to the accuracy, we set-up a controlled environment where we segment the data using the supervised method and sample the training, development and test sets so that they do not contain any OOVs. We also compare the performance of the method presented in Section 4.2, and BPE-based segmentation on the same data sets, and the case without segmentation. In order to achieve a fair comparison between the two vocabulary reduction methods, we train the splitting rules of our method and BPE only on the source side of the parallel data. In the second set of experiments, we evaluate our method in a real case scenario. We do not include the supervised method in this phase as its performance would be highly affected by the amount of OOVs in the training and test sets. In Experiment 2.a, we use data sets of 


\begin{tabular}{ccccc}
\hline Data set & Experiment & \#sentences (K) & \#tokens (M) & \#types (K) \\
\hline \hline TED & $(1)$ & 115 & $1.6(\mathrm{TR})-2.2(\mathrm{EN})$ & $141(\mathrm{TR})-44(\mathrm{EN})$ \\
TED & $(2 . \mathrm{a})$ & 133 & $1.9(\mathrm{TR})-2.7(\mathrm{EN})$ & $169(\mathrm{TR})-53(\mathrm{EN})$ \\
TED + Generic & $(2 . \mathrm{b})$ & 283 & $4.1(\mathrm{TR})-5.6(\mathrm{EN})$ & $268(\mathrm{TR})-96 \mathrm{~K}(\mathrm{EN})$ \\
\hline
\end{tabular}

Table 4. Data sets used in each experiment. $K$ - thousand, $M$ - million.

similar distribution, whereas in Experiment 2.b, we increase data sparsity by adding generic data to the training set. We segment the source side of parallel corpora using different methods while we segment the target side with BPE. We measure the performance in either experiment (2.a and 2.b) on the same test set.

We use two sets of data for training our NMT systems. The first data set is the Turkish-English portion of TED Talks (Cettolo et al., 2012) from IWSLT (Paul et al., 2010) and is used in Experiment 1 and 2.a. The second data set is a combination of TED Talks and a collection of generic data from EU Bookshop (Skadinš et al., 2014), Global Voices, Gnome, Tatoeba, Ubuntu (Tiedemann, 2012), KDE4 (Tiedemann, 2009), Open Subtitles (Lison and Tiedemann, 2016) and SETIMES (Tyers and Alperen, 2010), filtered using the invitation model of Cuong and Simaan (2014) to reduce the size. The generic data is used in Experiment 2.b. In all the experiments, we use development and test sets of 1,000 sentences and use the remaining data for training the models. The statistics of all the data sets used in each experiment are given in Table 4 .

The NMT models used in the evaluation are based on the Nematus toolkit (Sennrich et al., 2017). They have a hidden layer and embedding dimension of 1024, a mini-batch size of 100 and a learning rate of 0.01 . The dictionary size is 40,000 (src $\& \operatorname{trg})$ in the $1^{\text {st }}$, and $30,000(s r c)-40,000(\operatorname{trg})$ in the $2^{\text {nd }}$ experiment. We train the models using the Adagrad (Duchi et al., 2011) optimizer with a dropout rate of 0.1 (src \& trg) and 0.2 (embeddings and hidden layers). We shuffle the data at each epoch. BPE merge rules are of equal size to the dictionary. We train the models for 50 epochs and choose the best model on the development set for translating the test set.

The modified Morfessor FlatCat models (Grönroos et al., 2014) are trained with a perplexity threshold of 10, a length threshold of 5, and an output lexicon size of 40,000 (Experiment 1 \& 2.a) and 30,000 (Experiment 2.b), which is a new input parameter added to the model implementation. Training time is 20 minutes (using an Intel Xeon E31240 v5 CPU), while segmentation time varies from 10 to 30 minutes, depending on the corpus size. Performance is measured using the BLEU (Papineni et al., 2002), TER (Snover et al., 2006) and CHRF3 (Popovic, 2015) scores and significance tests are computed with Multeval (Clark et al., 2011). 
1. TED corpus, no-OOV case, voc $=40 \mathrm{~K}$

\begin{tabular}{cccc}
\hline \hline Method & BLEU $\uparrow$ & TER $\downarrow$ & CHRF3 $\uparrow$ \\
\hline No Segmentation & 17.77 & 68.07 & 38.94 \\
\hline BPE & 19.52 & 66.23 & 42.33 \\
\hline Supervised & $21.61^{\mathbf{\Lambda}}$ & $61.76^{\mathbf{\Lambda}}$ & $\mathbf{4 4 . 0 1}$ \\
\hline LMVR & $\mathbf{2 1 . 7 1}^{\mathbf{\Lambda}}$ & $\mathbf{6 1 . 4 1}^{\mathbf{\Lambda}}$ & 43.90
\end{tabular}

\begin{tabular}{cccc}
\hline Input (Reference) & Method & Segmentation & Output \\
\hline \hline ağlarını & BPE & ağ@@ larını & the cry \\
(the nets) & LMVR & ağ +ların & the nets \\
& Supervised & ağ +Noun + A3pl <EOW $>$ & networks \\
\hline ağlamayacak & BPE & ağ@@ lamayacak & will not survive \\
(would not be crying) & LMVR & ağlama +yacak & will not cry \\
& Supervised & ağla +Neg +Fut +A3sg <EW $>$ & will not cry \\
\hline
\end{tabular}

Table 5. Results of Experiment 1 - TED corpus and no-OOV case. Top: Output accuracies, where ${ }^{\boldsymbol{\Delta}}$ indicates statistically significant improvement over the BPE baseline (p-value $<0.05)$. Bottom: Translation examples.

\section{Results and Discussion}

Table 5 shows the performance of different segmentation methods in Experiment 1. Our linguistically motivated vocabulary reduction (LMVR) method achieves the best performance on average, proving our hypothesis that a correct morphological representation generates more accurate translations. Our method outperforms the strong baseline of BPE-based segmentation by 2.2 BLEU, 4.8 TER and 1.6 CHR3F points. The performance is slightly higher than the supervised method, which is related to the ambiguity caused by loss of information during the morphological analysis. The predicted vocabularies also indicate the significant difference between LVMR and BPE, where $73 \%$ of the sub-word units in the vocabulary are completely different. In order to better illustrate the properties of the generated sub-word units, we present example translations of two words from the test set. The two words have different roots, the first one is $a \breve{g}$ (translation: net), and the second one is ăgla (translation: (to) cry). BPE segments both words to the same root $a \breve{g}$, a character sequence frequently observed in root words in Turkish. In the first case, both unsupervised methods segment the word into the same sub-word units, while the embedding of the sub-word unit segmented with BPE is semantically ambiguous and generates unreliable translations. On the other hand, our method can preserve the correct meaning in both cases.

In Experiment 2, we evaluate our method at different rates of vocabulary reduction according to the vocabulary sizes given in Table 4 . All metrics confirm that our method achieves better performance than the baseline in both experiments. In Experiment 2.a, at a vocabulary reduction rate of 4.25 (140K $->40 \mathrm{~K})$, we obtain an improvement of 2.3 BLEU points over the baseline. In the most challenging case, Experiment 


\begin{tabular}{ccccccc}
\hline \multicolumn{3}{c}{ 2.a TED corpus, OOV case, voc $=$ 40K } & \multicolumn{3}{c}{ 2.b Large corpus, OOV case, voc=30K } \\
\hline \hline Method & BLEU $\uparrow$ & TER $\downarrow$ & CHRF3 $\uparrow$ & BLEU $\uparrow$ & TER $\downarrow$ & CHRF3 $\uparrow$ \\
\hline BPE & 20.45 & 64.50 & 42.65 & 24.42 & 60.14 & 47.05 \\
\hline LMVR & $\mathbf{2 2 . 7 6}^{\mathbf{\Lambda}}$ & $\mathbf{6 2 . 9 4}^{\mathbf{\Lambda}}$ & $\mathbf{4 5 . 3 6}$ & $\mathbf{2 5 . 4 2}^{\mathbf{\Lambda}}$ & $\mathbf{5 8 . 8 8}^{\mathbf{\Lambda}}$ & $\mathbf{4 7 . 7 1}$ \\
\hline
\end{tabular}

Table 6. Results of Experiment 2 - OOV presence and different rates of vocabulary reduction. ${ }^{\boldsymbol{\Delta}}$ indicates statistically significant improvement over the BPE baseline ( $p$-value $<0.05)$.

2.b, we increase the training set using data coming from varying domains, which maximizes the sparseness due to rare word forms in the corpus. Furthermore, we decrease the source vocabulary limit to 30,000 , requiring a vocabulary reduction rate of 9 (270K $\rightarrow 30 \mathrm{~K})$. As given in Table 6 , our method can still outperform the baseline by 1.0 BLEU point. The results and the computational efficiency of our method prove that it can be deployed in practical NMT systems trained with generic corpora.

\section{Conclusion}

In this paper we have addressed the vocabulary limitation in NMT, which has been an open issue in the translation of morphologically rich languages. For this purpose, we have proposed a novel linguistically motivated vocabulary reduction method that can achieve open vocabulary translation while, unlike previous approaches, maintaining a linguistic notion at the sub-word level. The method is completely unsupervised and can estimate a fixed size dictionary of sub-word units considering their individual morphological properties. We have evaluated our method against a statistical vocabulary reduction method and showed that our method obtains significantly better performance due to bringing a linguistic notion into the segmentation process.

\section{Acknowledgements}

This work has been partially supported by the EC-funded H2020 projects QT21 (grant no. 645452) and ModernMT (grant no. 645487). The authors would like to thank Arianna Bisazza and Prashant Mathur for their contributions to this study.

\section{Bibliography}

Bahdanau, Dzmitry, Kyunghyun Cho, and Yoshua Bengio. Neural machine translation by jointly learning to align and translate. CoRR, abs/1409.0473, 2014.

Bisazza, Arianna and Marcello Federico. Morphological pre-processing for Turkish to English statistical machine translation. In IWSLT, pages 129-135, 2009.

Bradbury, James and Richard Socher. MetaMind neural machine translation system for WMT 2016. In Proceedings of the 1st Conference on Machine Translation. ACL, 2016. 
Cettolo, Mauro, Christian Girardi, and Marcello Federico. WIT3: Web Inventory of Transcribed and Translated Talks. In Proceedings of EAMT, pages 261-268, 2012.

Cho, Kyunghyun, Bart van Merrienboer, Dzmitry Bahdanau, and Yoshua Bengio. On the Properties of Neural Machine Translation: Encoder-Decoder Approaches. In Proceedings of the 8th Workshop on Syntax, Semantics and Structure in Statistical Translation, pages 103-111, 2014.

Clark, Jonathan H., Chris Dyer, Alon Lavie, and Noah A. Smith. Better Hypothesis Testing for Statistical Machine Translation: Controlling for Optimizer Instability. In Proceedings of the 49th Annual Meeting of ACL, pages 176-181. ACL, 2011.

Creutz, Mathias and Krista Lagus. Inducing the morphological lexicon of a natural language from unannotated text. In Proceedings of the International and Interdisciplinary Conference on Adaptive Knowledge Representation and Reasoning, pages 51-59, 2005a.

Creutz, Mathias and Krista Lagus. Unsupervised morpheme segmentation and morphology induction from text corpora using Morfessor 1.0. Helsinki University of Technology, 2005b.

Creutz, Mathias and Krista Lagus. Unsupervised models for morpheme segmentation and morphology learning. Transactions on Speech and Language Processing, 4(1):3, 2007.

Cuong, Hoang and Khalil Simaan. Latent domain translation models in mix-of-domains haystack. In Proceedings of COLING, pages 1928-1939, 2014.

Duchi, John, Elad Hazan, and Yoram Singer. Adaptive subgradient methods for online learning and stochastic optimization. Journal of Machine Learning Research, 12(Jul):2121-2159, 2011.

Gage, Philip. A new algorithm for data compression. The C Users Journal, 12(2):23-38, 1994.

Grönroos, Stig-Arne, Sami Virpioja, Peter Smit, and Mikko Kurimo. Morfessor FlatCat: An HMM-Based Method for Unsupervised and Semi-Supervised Learning of Morphology. In COLING, pages 1177-1185, 2014.

Lee, Jason, Kyunghyun Cho, and Thomas Hofmann. Fully Character-Level Neural Machine Translation without Explicit Segmentation. CoRR, abs/1610.03017, 2016.

Ling, Wang, Isabel Trancoso, Chris Dyer, and Alan W Black. Character-based neural machine translation. CoRR, abs/1511.04586, 2015.

Lison, Pierre and Jörg Tiedemann. Opensubtitles2016: Extracting large parallel corpora from movie and tv subtitles. In Proceedings of LREC, 2016.

Luong, Minh-Thang and Christopher D Manning. Achieving open vocabulary neural machine translation with hybrid word-character models. In Proceedings of the 54th Annual Meeting of ACL. ACL, 2016.

Oflazer, Kemal. Two-level description of Turkish morphology. Literary and linguistic computing, 9(2):137-148, 1994.

Oflazer, Kemal and Ilknur Durgar El-Kahlout. Exploring different representational units in English-to-Turkish statistical machine translation. In Proceedings of the $2 n d$ Workshop on Statistical Machine Translation, pages 25-32. ACL, 2007.

Papineni, Kishore, Salim Roukos, Todd Ward, and Wei-Jing Zhu. BLEU: A Method for Automatic Evaluation of Machine Translation. In Proceedings of the 40th Annual Meeting of ACL, pages 311-318. ACL, 2002. 
Paul, Michael, Marcello Federico, and Sebastian Stücker. Overview of the IWSLT 2010 Evaluation Campaign. In Proceedings of IWSLT, pages 3-27, 2010.

Popovic, Maja. chrF: character n-gram F-score for automatic MT evaluation. 2015.

Sak, Haşim, Tunga Güngör, and Murat Saraçlar. Morphological disambiguation of Turkish text with perceptron algorithm. In International Conference on Intelligent Text Processing and Computational Linguistics, pages 107-118. Springer, 2007.

Sánchez-Cartagena, Victor M and Antonio Toral. Abu-MaTran at WMT 2016 Translation Task: Deep Learning, Morphological Segmentation and Tuning on Character Sequences. In Proceedings of the 1st Conference on Machine Translation. ACL, 2016.

Sennrich, Rico, Barry Haddow, and Alexandra Birch. Neural machine translation of rare words with subword units. Proceedings of the 54th Annual Meeting of the Association for Computational Linguistics, 2016.

Sennrich, Rico, Orhan Firat, Kyunghyun Cho, Alexandra Birch, Barry Haddow, Julian Hitschler, Marcin Junczys-Dowmunt, Samuel L"aubli, Antonio Valerio Miceli Barone, Jozef Mokry, and Maria Nadejde. Nematus: a Toolkit for Neural Machine Translation. In Proceedings of EACL, 2017.

Skadiňš, Raivis, Jörg Tiedemann, Roberts Rozis, and Daiga Deksne. Billions of Parallel Words for Free: Building and Using the EU Bookshop Corpus. In Proceedings of LREC. European Language Resources Association, 2014.

Snover, Matthew, Bonnie Dorr, Richard Schwartz, Linnea Micciulla, and Ralph Weischedel. A Study of Translation Error Rate with Targeted Human Annotation. In Proceedings of AMTA, 2006.

Sutskever, Ilya, Oriol Vinyals, and Quoc V Le. Sequence to sequence learning with neural networks. In Advances in neural information processing systems, pages 3104-3112, 2014.

Tiedemann, Jörg. News from OPUS-A collection of multilingual parallel corpora with tools and interfaces. In Recent advances in natural language processing, volume 5, pages 237-248, 2009.

Tiedemann, Jörg. Parallel Data, Tools and Interfaces in OPUS. In Proceedings of LREC. European Language Resources Association, 2012.

Tyers, Francis M and Murat Serdar Alperen. South-east European Eimes: A parallel corpus of balkan languages. In Proceedings of the LREC Workshop on Exploitation of Multilingual Resources and Tools for Central and (South-) Eastern European Languages, pages 49-53, 2010.

Wu, Yonghui, Mike Schuster, Zhifeng Chen, Quoc V Le, Mohammad Norouzi, Wolfgang Macherey, Maxim Krikun, Yuan Cao, Qin Gao, Klaus Macherey, et al. Google's Neural Machine Translation System: Bridging the Gap between Human and Machine Translation. CoRR, abs/1609.08144, 2016.

\section{Address for correspondence: \\ Duygu Ataman \\ ataman@fbk.eu \\ Via Sommarive 18, Povo, 38123 Trento, Italy}

